\title{
Structural and molecular basis for foot-and- mouth disease virus neutralization by two potent protective antibodies
}

\section{Dear Editor,}

Foot-and-mouth disease (FMD) is an economically devastating and highly contagious viral disease of cloven-hoofed animals with a global distribution. The causative agent, FMD virus (FMDV) is a small non-enveloped RNA virus, belonging to the Aphthoviruses genus within Picornaviridae family (Tuthill et al., 2010). Control of FMD has been largely reliant on vaccinations with inactivated virus vaccines. However, significant antigenic diversity within FMDV serotypes and inability of the vaccines to induce immune protection for a long duration of time impinge on the efficacy of available vaccines. The roles of neutralizing antibodies (NAbs) as the principal protective components of the immune responses to FMDV vaccination or infection have been well established (Pay and Hingley, 1987; Juleff et al., 2009). Passive immunization of NAbs has also been demonstrated to be effective in curing FMD and many viral diseases (Harmsen et al., 2007; Qiu et al., 2018). A deep understanding of the molecular basis for viral neutralization by antibodies and the identification of key viral epitopes would aid in the development of potent rationally designed broad-spectrum vaccine.

By using phage display immune libraries derived from four llamas, we previously identified 24 single-domain antibodies capable of neutralizing FMDV type $\mathrm{O}$ in vitro (Harmsen et al., 2007). Among these, M8 and M170 exhibited relatively strong neutralizing activities. To investigate the serotype specificity or cross-reactivity of M8 and M170, we propagated and purified FMDV O, A, Asia 1 as well as C serotype strains and separately examined their binding abilities to each antibody by enzyme-linked immunosorbent assay (ELISA). The ELISA results revealed that M170 only binds to type $\mathrm{O}$, but $\mathrm{M} 8$ is capable of reacting with all the indicated serotypes, suggesting that M8 and M170 are type cross-reactive and O-specific, respectively (Fig. S1A). Surface plasmon resonance (SPR) experiments verified that both $\mathrm{M} 8$ and $\mathrm{M} 170$ interact with type $\mathrm{O}$ with a high binding affinity of $0.5 \mathrm{nmol} / \mathrm{L}$ and $1.0 \mathrm{nmol} / \mathrm{L}$, respectively (Fig. $1 \mathrm{~A}$ ). To explore whether these two antibodies can simultaneously bind the virus, we performed a competitive SPR assay and the results indicated that the binding of one antibody blocks the attachment of the other (Fig. 1B), which suggests that M8 and M170 compete with each other for simultaneous binding albeit with different characteristics in binding distinct serotypes and targeting distinct antigenic sites. Cell-based neutralization assays showed that both M8 and M170 exhibit potent neutralizing activities against type $O$ with a $50 \%$ neutralizing concentration value ( Neut $_{50}$ ) of 0.8 and 3.2 $\mu \mathrm{mol} / \mathrm{L}$, respectively (Fig. 1C, up). Perhaps correlated with the inability of simultaneous binding, the cocktail of M8 and M170 did not exhibit synergistic neutralization activity (Fig. 1C, down). Interestingly, results of a fluorescencebased assay revealed that M8, rather than M170, destabilized FMDV particles by $3-8{ }^{\circ} \mathrm{C}$ in an incubation time dependent manner at physiological conditions $(\mathrm{pH} 7.5)$, indicating that physical destabilization of viral particles that interferes with normal uncoating may be a possible neutralization mechanism for M8 (Fig. S1B).

Given the potent neutralizing activities of M8 and M170 at sub- $\mu \mathrm{mol} / \mathrm{L}$ concentrations, we next sought to assess the in vivo protection efficacy of these two antibodies in Guinea pigs (GP). Guinea pigs were administered M8 or M170 1 day before or after inoculation of the FMDV, representing a prophylactic $(P)$ or therapeutic $(T)$ setting, respectively (Fig. 1D). In the prophylactic mode, a single intramuscular injection of M8 at $2.5 \mathrm{mg} / \mathrm{kg}$ protected $75 \%$ of the animals from FMD until the end of the experiments and delayed morbidity in rest of the animals exhibiting symptoms of FMD when compared to the control. In contrast to this, only $25 \%$ of the animals receiving the same dose of M170 were protected from FMDV infections, while $75 \%$ of the animals developed FMD symptoms later at day $4 \mathrm{PI}$ (Fig. 1E, up). In line with these results, viral loads in the blood were nearly undetectable during the course of the monitoring of the infection in M8 administrated group. However, a severe viremia occurred at day $2 \mathrm{PI}$ in control group. Pre-treatment of M170 alleviated the viremia and cleared $\sim 95 \%$ of the viral titer when compared to the control group at day $3 \mathrm{PI}$ (Fig. 1E, down). Therapeutic administration of either M8 or M170 one day after virus challenge resulted in a relatively weak protective efficacy with $25 \%$ protection for M8 and delayed morbidity for M170 when compared to the control (Fig. 1F, 

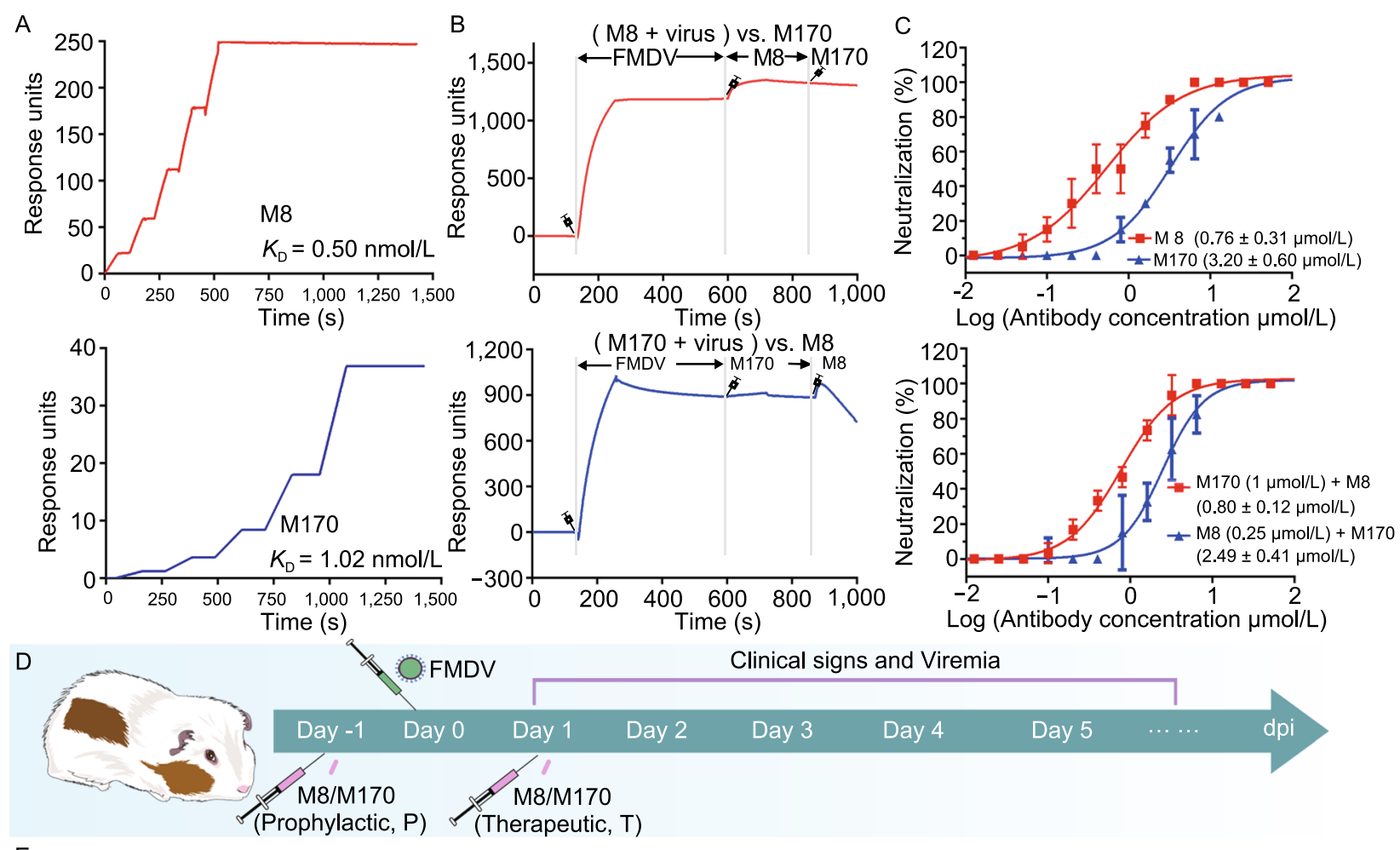

$\mathrm{E}$

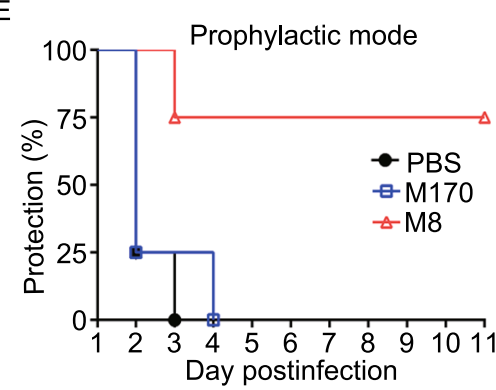

FMDV

Clinical signs and Viremia
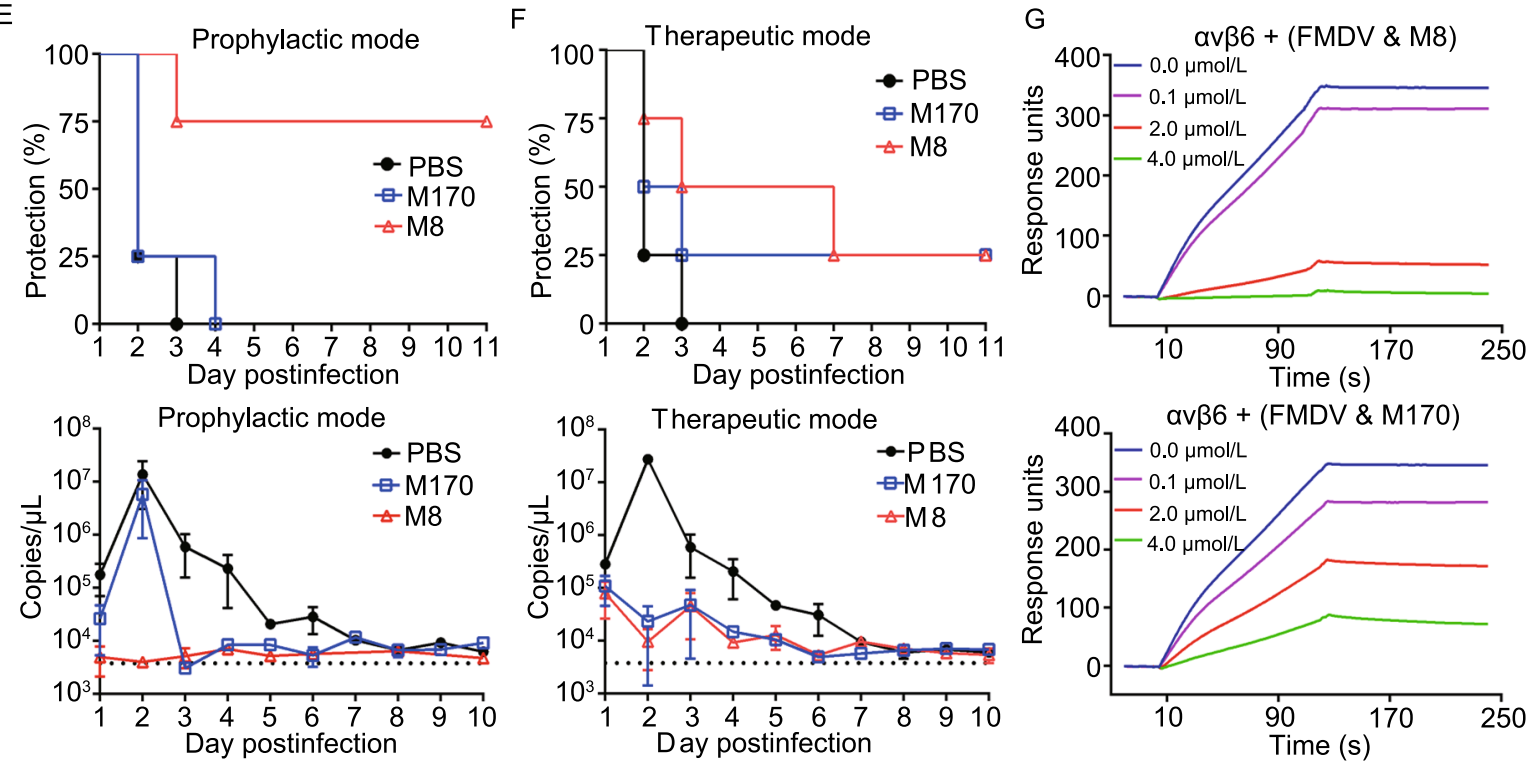

$\mathrm{H}$
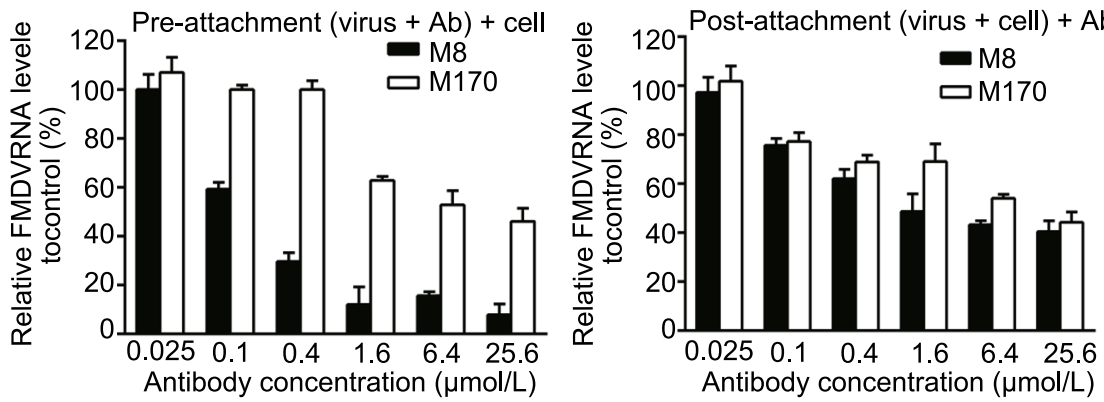
Figure 1. Characterizations of anti-FMDV NAbs M8 and M170. (A) BIAcore SPR kinetic profile of mAb M8 (top) and M170 (bottom) against FMDV $O$. The binding affinity is depicted by $K_{D}$ (equilibrium dissociation constant, $K_{\mathrm{D}}=K_{\mathrm{d}} / K_{\mathrm{a}}$ ), which was calculated by the BIAcore 3000 analysis software (BIAevaluation version 4.1). (B) Analysis of the simultaneous binding of $M 8$ and M170 to FMDV through a competitive SPR assay. Due to the requirement of acidic condition for the sensor-labeling, M8 or M170, rather than FMDV, was loaded onto the sensor. In the upper panel, the sensor was labeled with M8. FMDV was inject first, followed by the second injection of M8 again to fully occupy all binding sites for M8 on viral surface, then M170 flowed through. In the bottom panel, the sensor was labeled with M170. The SPR procedure of M170 is same to that of M8. Note: signals (during 800-1000 s) decreased upon M8 binding in the bottom panel, which might result from the ability that M8 has the potential to strip FMDV off the M170-labelled sensor due to the higher binding affinity. (C) Neutralization of FMDV $\mathrm{O}$ by M8/ M170 (top) and the combination of M8 and M170 (bottom) using plaque-reduction neutralization assay. Neut $_{50}$ values indicate concentration of antibody required to neutralize fifty percent of the viral titer. The Neut 50 of M8 and M170 were $0.8 \mu \mathrm{mol} / \mathrm{L}$ and $3.2 \mu \mathrm{mol} / \mathrm{L}$, respectively. The Data is presented as the mean \pm SD of triplicate measurements. (D) Schematic diagram of M8 or M170 treatment in guinea pig model. Groups of guinea pigs ( $n=$ 4) were administrated intramuscularly with M8/M170 (2.5 mg/ $\mathrm{kg}) 1$ day before (prophylactic) or after (therapeutic) challenge with $100 I D_{50}$ of FMDV on the left hind footpad. Guinea pigs injected intramuscularly with PBS before or after challenge were acted as control groups. The day of FMDV infection was marked as day 0 . Protection of guinea pigs against FMDV $O$ by passive immunization with M8 or M170 was analyzed in the prophylactic (Fig. 1E, up) and therapeutic (Fig. 1F, up) modes. No lesions on rear feet were considered as full protection. The copies of virus mRNA in the blood sample from guinea pigs of the prophylactic (Fig. 1E, down) and therapeutic (Fig. 1F, down) groups were quantified by the real-time quantitative PCR (RTqPCR), the limit of detection (LOD) of viral mRNA in blood was labeled. (G) BIAcore SPR kinetics shows the competitive binding of M8/M170 and $\alpha v \beta 6$ integrin to FMDV O. For both panels, av $\beta 6$ integrin was immobilized onto the sensor chips. Mixtures of FMDV O $(20 \mathrm{nmol} / \mathrm{L})$ with various concentrations of M8 (up) or M170 (down) acted as running phase to flow through the sensor. Binding signals were detected. $(\mathrm{H})$ Amount of virions remaining on the cell surface, as detected by real-time PCR, when exposed to M8 or M170 before or after the virions attach to BHK21 cells. Data is presented as the mean \pm SD. Experiments were repeated in triplicate.

up). Correlated with this, high levels of viral replication prior to therapeutic administration of the NAbs were detected, whereas M8 and M170 treatment at all indicated time-points resulted in largely reduced viral titers with a clear trend for greater reduction with earlier treatment (Fig. 1F, down). Of note, efficient viral infection that had been established at day
$1 \mathrm{PI}$ may require a higher dose of antibodies for rapid and complete clearance of FMDV or an earlier therapeutic treatment for suppressing massive replication of viruses.

Integrins, heterodimeric adhesive glycosylated membrane proteins located on the surface of most cells, have been identified as FMDV receptors that bind viruses via a small cleft at the subunit interface of the head (Kotecha et al., 2017). FMDV enters host cells by integrin-mediated endocytosis, then disassociates into pentamers to release RNA in the acidic environment of the endocytic compartments. To investigate whether M8 or M170 interferes with the binding of FMDV to integrin, we performed competitive SPR-based binding assays. The results indicated that both M8 and M170 can prevent FMDV from binding integrin in a concentrationdependent manner (Fig. 1G). Besides, the blocking of binding by M8 appeared more potent when compared to M170, which explains the differential neutralizing activities of these two antibodies (Fig. 1G). Consistent with the competitive binding assay results, in cell-based viral infection model, both M8 and M170 efficiently prevented FMDV type $O$ attachment to the cell surface and could displace the viral particles that had already bound to the cell surface in a dosedependent manner (Fig. 1H).

To define the key epitopes and atomic determinants of the interactions between FMDV and its two NAbs precisely, we determined the cryo-EM structures of FMDV type $O$ in complex with M8 or M170. Cryo-EM micrographs of the purified formaldehyde-inactivated FMDV mature particles in complex with M8 or M170 were recorded using an FEI Titan Krios electron microscope equipped with a Gatan K2 Summit detector (Fig. S2). Icosahedral reconstructions of FMDV-M8 and FMDV-M170 complexes were achieved at resolutions of $3.2 \AA$ and $3.1 \AA$, respectively (Fig. S3). The maps for the viral capsid were of sufficient resolution to build and refine the atomic model. But, the densities for antibodies and binding interface were relatively weak due to the unsaturated occupancy and conformational heterogeneity (Fig. S4), which is in line with the structural observations of flexible bindings of integrins to FMDV at different angles (Kotecha et al., 2017). To improve the resolution of the binding interface, local refinement by using an optimized "block-based" reconstruction approach (Wang et al., 2019) was performed. This led to an improvement of the local resolution to $3.9 \AA$ and $3.5 \AA$ for the binding interfaces of FMDV-M8 and FMDV-M170, respectively, enabling a reliable analysis of the interaction modes (Fig. S5).

As expected, M8 and M170 recognize distinct antigenic sites (Fig. 2). M8 binds to the FMDV viral surface within the pentameric building blocks surrounding the fivefold axis at a position akin to the "canyon" in enteroviruses (Dang et al., 2014). In contrast, M170 targets the surface along the edges of pentameric building block of the virus (Fig. 2A-D), nearby the twofold axis at a position similar to that observed for D6 antibody bound to EV71 (Zhu et al., 2018). Apart from targeting distinct antigenic sites, M8 and M170 adopt significantly different configurations upon binding to the FMDV 
A

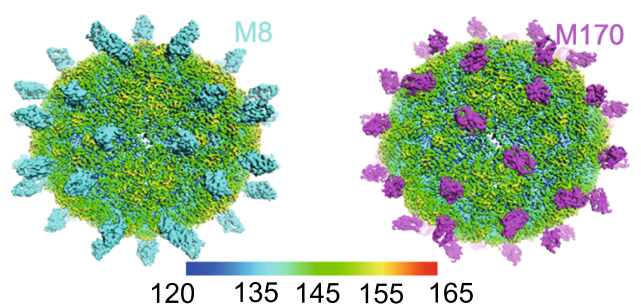

FMDV in complex with M8 FMDV in complex with M170

C

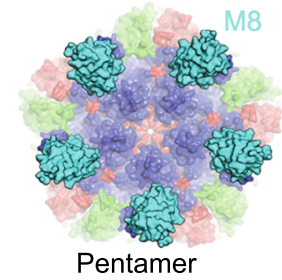

E

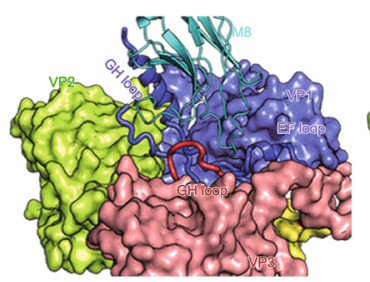

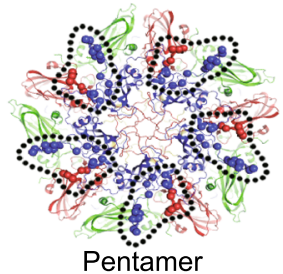

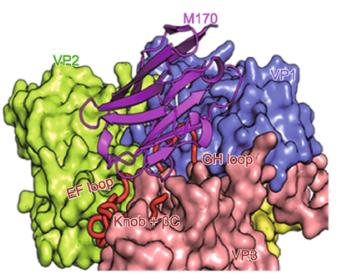

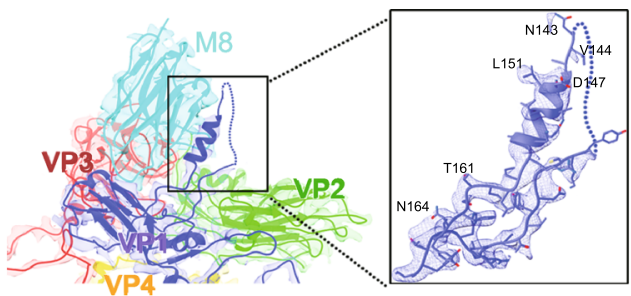

FMDV in complex with M8

VP1 GH loop
D

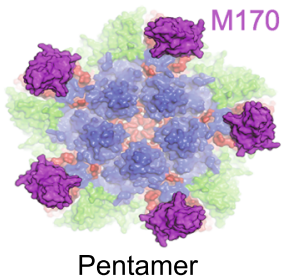

$\mathrm{F}$

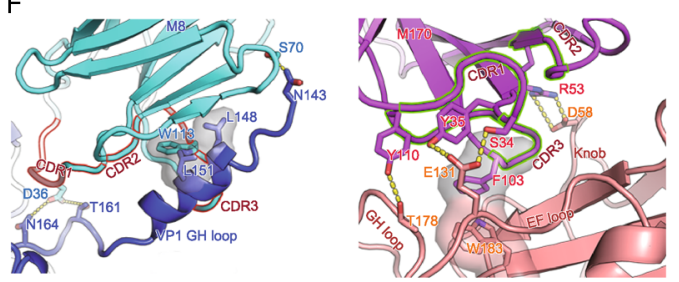

G
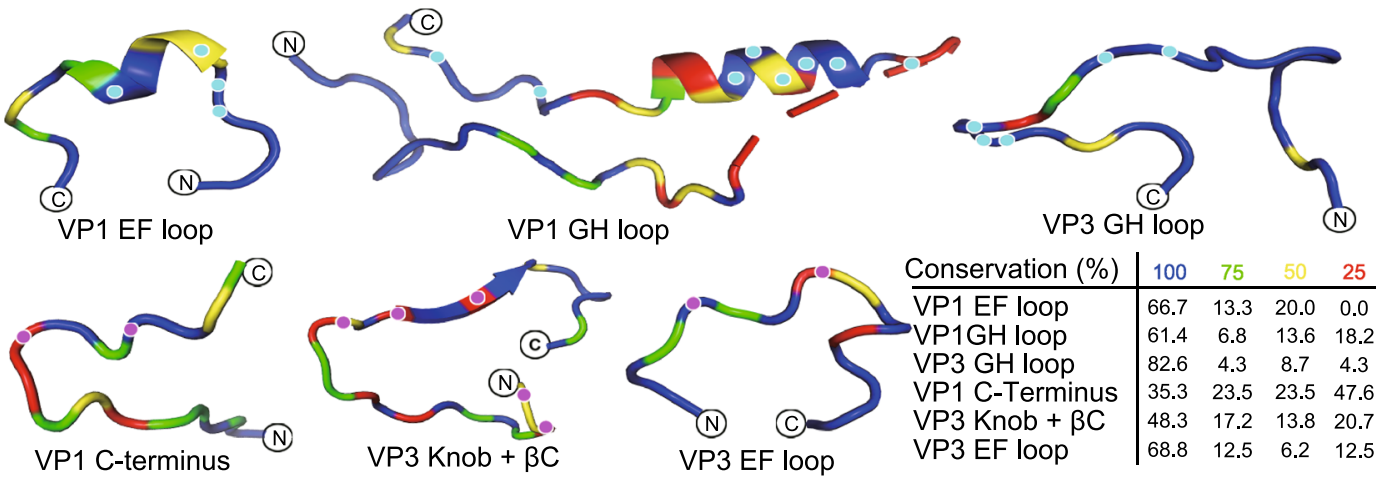

$\mathrm{H}$

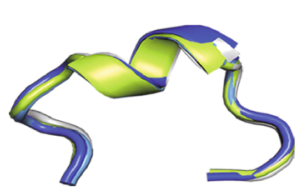

VP1 EF loop

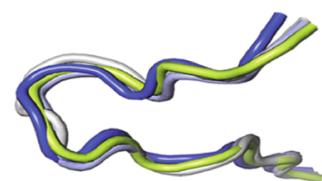

VP1 C-terminus

।
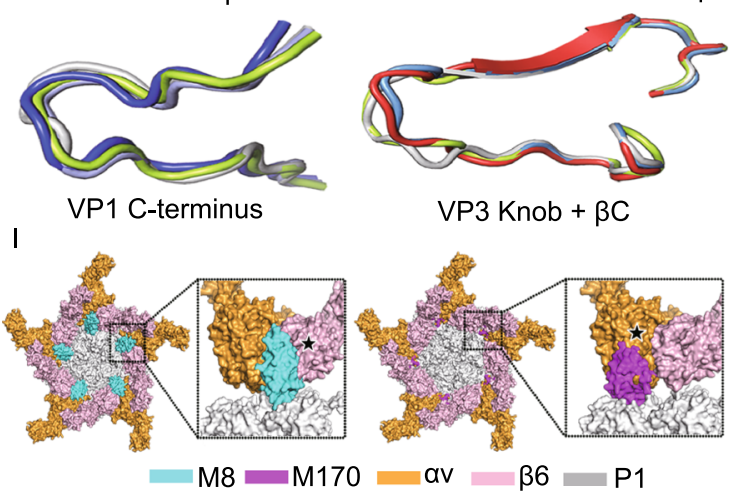
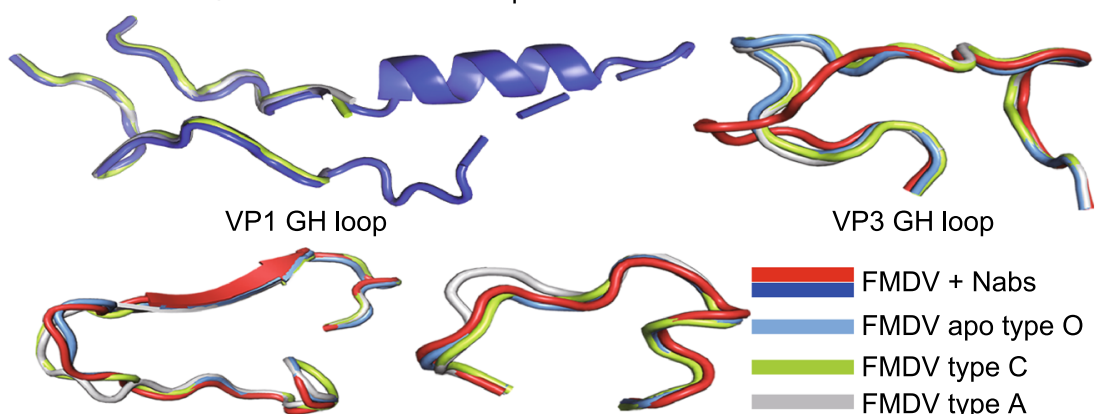

VP3 EF loop

J

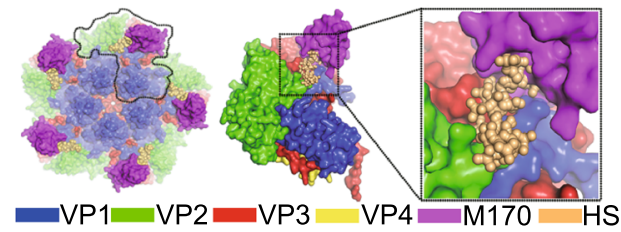

FMDV+M170 pentamer FMDV+M170 protomer 
Figure 2. Cryo-EM structures, mechanism of neutralization of FMDV by M8 or M170. (A) Cryo-EM maps of FMDV-M8-complex (left) and FMDV-M170-complex (right). The viral capsids of both complexes are rainbow-colored as the color bar shown below; the M8 and M170 are colored in cyan and magenta, respectively. (B) Electron density maps for the binding interface of M8 and one protomer of the FMDV capsid. The inset shows the density maps and atomic model of VP1 GH loop. Residues with side chains are labeled. Surface representation (left) and epitopes (right) of M8 (C) and M170 (D) on a viral pentamer. The pentamers are shown as surface (left) or cartoon (right) in the signature colors (VP1, blue; VP2, green; VP3, red), while $\mathrm{M} 8$ and M170 are colored in the same scheme as in Fig. 2A. The epitopes of M8 (right in 2C) and M170 (right in 2D) are shown as spheres and those from one protomeric unit are circled by black dotted lines. (E) The structural basis of M8 (left) and M170 (right) binding to FMDV. Loops from FMDV involved in interactions with M8/M170 are shown as cartoon, while the remaining parts are shown as surface. $M 8$ and $M 170$ are represented as cartoon. The color scheme is as in Fig. 2A. (F) Detailed interactions between FMDV and M8 (left), as well as FMDV and M170 (right). Residues involved in the binding are shown as sticks and hydrogen bonds are shown as yellow dashed lines. The complementary determining regions of M8 and M170 involved in the interaction with viral capsids are outlined in red and green, respectively. (G) Sequence conservation analysis. The loops from FMDV $O$ that are involved in interactions between FMDV and M8 (VP1 GH loop, VP1 EF loop, VP3 GH loop), as well as FMDV and M170 (VP1 C-terminus, VP3 Knob- $\beta C$ and VP3 EF loop) are aligned with those of 3 other FMDV serotypes (A, Asia 1 and $C$ ) and colored according to sequence conservation as listed in the table below. Residues involved in binding to M8 and M170 are marked by cyan and magenta dots. $(\mathrm{H})$ Structural conservation analysis. The same loops in $2 \mathrm{C}$ colored in the signature color scheme are superposed with their counterparts from the apo O (sky blue), A (gray) and C (lime). (I) Clashes between M8 (left)/M170 (right) and $\alpha v \beta 6$ receptor. Superimpositions of structures of the FMDVM8/M170 and FMDV- $\alpha$ $\beta 6$ are shown in pentamer. The capsid pentamer, M8, M170, and receptor subunits $\alpha \mathrm{v}$ and $\beta 6$ are colored in grey, cyan, magenta, bright orange and light pink, respectively. Clashes between M8/M170 and receptor subunits are prominent and marked with star symbols. (J) Clashes between M170 and HS cellular receptor. Color scheme for capsid pentamer and M170 is same as in Fig. 2D. HS receptor is shown as ball and colored in yellow. A protomer together with its corresponding binders (HS and M170) is marked by black lines. The inset shows the clashes between HS and M170.

(Fig. S6A). When viewed down the fivefold axis, individual M8 stands vertically, mimicking the association mode between the $\beta$ subunit of integrin and FMDV (Kotecha et al., 2017), while M170 locates aside M8, adjacent to the twofold axis, but inclines by $\sim 45^{\circ}$ towards the viral surface in an attachment mode resembling the strategy used by the $a$ subunit of integrin for its interaction with FMDV (Fig. S6A). However, minor steric clashes resulting from one overlapped residue within the binding sites and the inclined posture of M170 prevent the simultaneous binding of M8 and M170 to FMDV (Fig. S6B), thereby failing to make up a non-competing pair of antibody cocktail.

Analysis of the structures of FMDV in complex with M8 or M170 helped identify the epitopes of these two antibodies. M8 recognizes a conformational epitope constructed by VP1 EF, VP1 GH and VP3 GH loops within a protomer (Figs. 2E and S7). All three complementary determining regions (CDR): CDR1 (residues 32-39), CDR2 (residues 60) and CDR3 (residues 110-119) and framework region (FR, residues 66-70) of M8 are involved in extensive binding contacts with VP1 and VP3, burying $\sim 1,100 \AA^{2}$ of surface area by pinching the helix of VP1 GH loop in the "up" conformation between the CDR2 and CDR3 (Figs. 2B, 2F and S8). Notably, VP1 GH loop, highly disordered in the native virus structures, adopts a more ordered position lying along the viral surface when the disulfide bond linking the base of the loop (C134) to C130 of VP2 is reduced (Logan et al., 1993). The native high-resolution structural details of this loop enable us to define precisely the atomic determinants of the virus-antibody interaction. The epitope recognized by $\mathrm{M} 8$ primarily locates in the VP1 EF loop (A93, P94, V95 and L98), VP1 GH loop (K133, N143, V144, D147, L148, Q149, L151, A152, Q153, T161, N164 and A167), and the VP3 GH loop (A171, S172, A175, E176 and T177) (Fig. 2E). When compared to M8, M170 primarily binds to VP3 via recognizing the "knob", $\beta C, B C, C D, E F$ and GH loops of VP3, covering most of the exposed surface of VP3, and VP1 C-terminus (Figs. 2E and S7), which is way far beyond the previously reported antigenic site 4 within VP3 (McCahon et al., 1989). The M170 paratope is composed of CDR1 (residues 30-35), CDR2 (residues 53-56) and CDR3 (residues 103-110), which bury a surface area of about $\sim 900 \AA^{2}$ at the interface with VP3 and VP1 through hydrophilic interactions and hydrophobic contacts (Fig. 2E and $2 F$ ). The epitope of M170 mainly includes residues $57-59$ of the $\beta B$ "knob", residues 71 and 73 of $B C$ loop, residue 76 of $\beta C$, residues 78 and 85 of $C D$ loop, residues 131 and 134 of EF loop, residues 177, 178 and 183 of GH loop in VP3 and residues 199-202 of VP1 C-terminus (Fig. 2F). Tight binding between the antibodies and FMDV is accomplished by forming extensive hydrophilic interactions, including hydrogen bonds and salt bridges (Tables S2 and S3).

Many NAbs with potent neutralizing activities are virus specific. Cross-reactive NAbs, in general, exhibit relatively weak antiviral activities. By contrast, in our case, M8, capable of cross-binding to several FMDV serotypes, including $\mathrm{O}, \mathrm{A}$, Asia 1 and $\mathrm{C}$ strains, shows better neutralizing activity than M170 that is a type O-specific antibody (Figs. S1 and 1C). More importantly, cross-reactive NAbs, such as M8, primarily targeting more conserved epitopes offer clues for identifying the Achilles' heel of viruses between serotypes, even between genera, since these regions can be targeted 
for the development of antiviral drugs or broad-spectrum vaccine design. To decipher the structural basis for serotypespecific and cross-reactive binding of $M 8$ and $M 170$, sequence and structural alignments of these four FMDV serotypes focusing on key epitopes were performed. As a major antigenic site, VP1 GH loop bears, to some extent, sequence and structural diversity within FMDV serotypes. Therefore, VP1 GH loop together with other antigenic sites can be used to distinguish type $O$ from other serotypes. However, this region also contains a conformationally conserved helix (disordered in the native structures) with a conserved RGD motif within serotypes for receptor binding (Fig. $2 \mathrm{G}$ and $2 \mathrm{H}$ ). Notably, $\sim 70 \%$ of the binding interface between M8 and VP1 GH loop is made up by conserved residues among serotypes. Incidentally, VP1 EF and VP3 $\mathrm{GH}$ loops exhibit even higher conservation both in sequence and configuration (Fig. $2 \mathrm{G}$ and $2 \mathrm{H}$ ). Interestingly, VP3 $\mathrm{GH}$, rather than other loops, adopts an alternative configuration upon M8 binding, whilst the binding of M170 does not mediate any notable conformational change although the NAb does target VP3 GH loop as well (Fig. S9). Overall, out of the 21 residues in the M8 epitope, $15(\sim 72 \%)$ are identical among these four serotypes (Fig. S7), which explains the cross-reactivity and comparable binding affinities. In contrast to M8, M170 buries $\sim 680 \AA^{2}$ of the VP3 surface by interaction with the "knob", $B C, \beta C, C D, E F$ and $\mathrm{GH}$ loops as well as $\sim 220 \AA^{2}$ of VP1 via association with its C-terminal loop. Except for VP3 GH loop, subtle conformational variations in the binding areas for M170 are observed within these four serotypes and most residues necessary for M170 binding are not conserved (Fig. $2 \mathrm{G}$ and $2 \mathrm{H}$ ). The specificity of the targeted region both in sequence and configuration explain the serotype-specificity of M170. In addition, residues comprising the epitopes of M170 are highly conserved across currently circulating type $\mathrm{O}$ isolates, suggesting that M170 is likely capable of neutralizing most FMDV type $O$ strains (Fig. S10).

In addition to integrin, FMDV can often readily adapt to tissue culture, where infection occurs in the absence of integrin via acquired basic mutations that confer an ability on the virus to interact with heparin sulfate (HS) (SaCarvalho et al., 1997). Cryo-EM structures of FMDV type $O$ in complex with $\alpha v \beta 6$, hitherto not at high resolution, reveal that the fully open form of the integrin attaches to an extended $\mathrm{GH}$ loop of VP1 via interactions with the RGD motif plus downstream hydrophobic residues and a conserved previously identified $\mathrm{HS}$ binding site through an N-linked sugar (Kotecha et al., 2017). Superposition of the FMDV-M8/FMDV-M170 and FMDV- $\alpha$ $\beta 6$ complex structures revealed clashes between the two subunits of $\alpha v \beta 6$ and M8/M170 (Fig. 2I). Notably, the structural overlay analysis reveals that M8 competes with $\beta 6$ subunit for binding to the RGD motif in a similar interaction mode and M170 partially occupies the av subunit binding site on the virus, where an $\mathrm{N}$-linked sugar from the av is oriented (Fig. 2l). Thereby, binding of M8 or M170 can completely block the attachment of integrin to FMDV owing to the substantially stronger binding affinities of M8 or M170 for FMDV when compared to that of the integrin. In addition, M170 targets the HS binding site, occluding the infection mediated by sulfated sugars as well (Fig. 2J). Notably, M8 and M170 recognize the RGD motif and the HS binding site, respectively, having great potential to occlude viral mutational escape. Footprints of $\alpha v \beta 6, \mathrm{HS}, \mathrm{M} 8$ and M170 on the FMDV surface reveal overlapped patches with areas of $\sim 2,800 \AA^{2}, \sim 1,900 \AA^{2}$ and $\sim 120 \AA^{2}$, between $\alpha \mathrm{v} \beta 6$ and M8/ $M 170$, and between HS and M170, respectively (Figs. S6C and 6D). Collectively, the abilities of M8 and M170 in preventing FMDV from attaching to host cell surface can be attributed to steric clashes arising out of partially overlapping binding sites.

For FMDV, the intact virions with a sedimentation coefficient of $146 \mathrm{~S}$ can irreversibly dissociate into stable pentamers, referred to as $12 \mathrm{~S}$ viral capsid degradation products, leading to dramatically decreased immunogenic potency of these vaccines (Paton et al., 2009). It is therefore critical to quantify and continuously monitor the $146 \mathrm{~S}$ particles present in the crude FMDV antigen preparations used for vaccine manufacturing. A prerequisite to the development of ELISAbased tests for the quality control of FMD vaccines is the availability of antibodies that can specifically detect $146 \mathrm{~S}$ particles. Antibodies that specifically bind to $146 \mathrm{~S}$ particles are rarely reported because most of the mAbs can bind to both $146 S$ and $12 S$ particles. In our previous study we reported that M170 specifically interacts with $146 \mathrm{~S}$ particles, and cannot bind 12S particles directly (Fig. S11) (Harmsen et al., 2011). During the de-assembly of the $146 \mathrm{~S}$ particle into $12 S$ pentamers, the capsid undergoes significant conformational rearrangements accompanied by a $\sim 4.6^{\circ}$ rotation of the bulk of VP3 towards the periphery, giving rise to a structural shift of some distal loops, including EF, HI, DE and $\mathrm{BC}$ loops, in the range of 4-9 $\AA$ (Fig. S11). Remarkably and in line with the inability of M170 to recognize $12 \mathrm{~S}$ particles, some residues of the M170 epitope, such as D71 and V73 in $\mathrm{BC}$ loop, E131 and K134 in EF loop, lie in the region with the largest conformational changes (Fig. S11). In contrast to M170, M8 shows indistinguishable binding activities against $146 \mathrm{~S}$ and $12 \mathrm{~S}$ since its epitope is located beyond this region (Fig. S11). Therefore, the 146S-specific M170, together with 12S-directed antibody, can be combined to develop an ELISA system for the quantification of $146 \mathrm{~S}$ particles during vaccine manufacturing. The molecular features of the M8 and M170 epitopes unveiled in this study pose interesting targets for structure-based rational broad-spectrum vaccine design and effective antigen detection development for use in vaccine quality control. Our studies also highlight the promise of simultaneous administration of vaccines and 
therapeutic antibodies for rapid and complete protection prior to the establishment of effective immune responses elicited by FMDV vaccines.

\section{FOOTNOTES}

Methods, including statements of data availability and any associated accession codes and references, are available in the supplementary materials.

We thank B. Zhu, X. Huang and G. Ji for Cryo-EM data collection at the Center for Biological imaging (CBI), Institute of Biophysics, and Y. Chen, Z. Yang and B. Zhou for SPR technical support and guidance. We also thank P. Du, X.Y Zhi, Y. Zhang for their guidance in preparation and purification of virus and antibody, and S.H Yin, J.X $\mathrm{Ru}$ for their help and guidance in guinea pigs experiments.

This work was supported by the project National Key Research and Development Program (Nos. 2017YFD05000900, 2018YFA0900801, 2017YFD0502200 and 2017YFD0502300), the National Natural Science Foundation of China (No. 31941011, 12034006, 31672592, 31873023, 31811540395, 31772739, 315725153 and 1811540395) and the Strategic Priority Research Program (XDB29010000). Xiangxi Wang was supported by Ten Thousand Talent Program and the NSFS Innovative Research Group (No. 81921005).

Hu Dong, Pan Liu, Manyuan Bai, Kang Wang, Rui Feng, Dandan Zhu, Yao Sun, Suyu Mu, Haozhou Li, Michiel Harmsen, Shiqi Sun, Xiangxi Wang and Huichen Guo declare that they have no conflict of interest.

Animal experiments were carried out in accordance with the regulations for the administration of affairs concerning experimental animals approved by the State Science and Technology Commission of the People's Republic of China and by the Committee for Animal Welfare and Safety in the Lanzhou Veterinary Research Institute of the Chinese Academy of Agricultural Sciences (No. LVRIAEC2018-008).

The cryo-EM density maps of icosahedral reconstructions for FMDV-M8 and FMDV-M170 and blocked reconstructions for the binding interface of FMDV-M8 and FMDV-M170 have been deposited in the Electron Microscopy Data Bank under accession codes: EMD-30843, EMD-30845, EMD-30844 and EMD-30846, respectively. The corresponding atomic coordinates have been submitted to the Protein Data Bank with accession numbers: 7DSS and 7DST, respectively.

H.D., P.L., and M.B. performed the experiments; P.L. and K.W. collected Cryo-EM data; P.L. and X.W. solved the structure; M.M. provided suggestion and some data about NAbs; D.Z. and R.F. did SPR experiments; S.M. and H.L help for doing some biological function verification experiment, S.S., X.W., and H.G. designed the study, H.D., S.S., and X.W. analyzed data, and H.D., X.W. wrote the manuscript, M.M., S.S., and H.G revised manuscript.

Hu Dong ${ }^{1}$, Pan Liu ${ }^{2,3}$, Manyuan Bai ${ }^{1}$, Kang Wang ${ }^{2}$, Rui Feng ${ }^{2}$, Dandan Zhu ${ }^{2}$, Yao Sun ${ }^{2}$, Suyu Mu ${ }^{1}$, Haozhou Li ${ }^{1}$, Michiel Harmsen ${ }^{4}$, Shiqi Sun ${ }^{1 凶}$, Xiangxi Wang ${ }^{2 \bowtie}$, Huichen Guo ${ }^{1 凶}$

\footnotetext{
${ }^{1}$ State Key Laboratory of Veterinary Etiological Biology and National
} Foot and Mouth Disease Reference Laboratory, Lanzhou
Veterinary Research Institute, Chinese Academy of Agricultural Sciences, Lanzhou 730046, China

2 CAS Key Laboratory of Infection and Immunity, CAS Center for Excellence in Biomacromolecules, Institute of Biophysics, Chinese Academy of Sciences, Beijing 100101, China

${ }^{3}$ University of Chinese Academy of Sciences, Beijing 100049, China

${ }^{4}$ Division Virology, Wageningen Bioveterinary Research, P.O. Box 65, 8200 AB Lelystad, The Netherlands

$\triangle$ Correspondence: sunshiqi@caas.cn (S. Sun), xiangxi@ibp.ac.cn (X. Wang), guohuichen@caas.cn (H. Guo)

Accepted December 30, 2020

\section{OPEN ACCESS}

This article is licensed under a Creative Commons Attribution 4.0 International License, which permits use, sharing, adaptation, distribution and reproduction in any medium or format, as long as you give appropriate credit to the original author(s) and the source, provide a link to the Creative Commons licence, and indicate if changes were made. The images or other third party material in this article are included in the article's Creative Commons licence, unless indicated otherwise in a credit line to the material. If material is not included in the article's Creative Commons licence and your intended use is not permitted by statutory regulation or exceeds the permitted use, you will need to obtain permission directly from the copyright holder. To view a copy of this licence, visit http:// creativecommons.org/licenses/by/4.0/.

\section{REFERENCES}

Dang M, Wang X, Wang Q, Wang Y, Lin J, Sun Y, Li X, Zhang L, Lou $Z$, Wang $J$ et al (2014) Molecular mechanism of SCARB2mediated attachment and uncoating of EV71. Protein \& Cell 5:692-703

Harmsen MM, van Solt CB, Fijten HP, van Keulen L, Rosalia RA, Weerdmeester K, Cornelissen AH, De Bruin MG, Eble PL, Dekker A (2007) Passive immunization of guinea pigs with llama single-domain antibody fragments against foot-and-mouth disease. Vet Microbiol 120:193-206

Harmsen MM, Fijten HPD, Westra DF, Coco-Martin JM (2011) Effect of thiomersal on dissociation of intact (146S) foot-and-mouth disease virions into $12 \mathrm{~S}$ particles as assessed by novel ELISAs specific for either $146 \mathrm{~S}$ or $12 \mathrm{~S}$ particles. Vaccine 29:2682-2690

Harmsen MM, Seago J, Perez E, Charleston B, Eble PL, Dekker A (2017) Isolation of single-domain antibody fragments that preferentially detect intact (146S) particles of foot-and-mouth disease virus for use in vaccine quality control. Front Immunol. https://doi. org/10.3389/fimmu.2017.00960

Kotecha A, Wang Q, Dong XC, Ilca SL, Ondiviela M, Zihe R, Seago J, Charleston B, Fry EE, Abrescia NGA, Springer TA, Huiskonen JT, Stuart DI (2017) Rules of engagement between av $\beta 6$ integrin and foot-and-mouth disease virus. Nat Commun 8:8

Logan D, Abu-Ghazaleh R, Blakemore W, Curry S, Jackson T, King A, Lea S, Lewis R, Newman J, Parry N et al (1993) Structure of a 
major immunogenic site on foot-and-mouth disease virus. Nature 362:566-568

Juleff N, Windsor M, Lefevre EA, Gubbins S, Hamblin P, Reid E, McLaughlin K, Beverley PCL, Morrison IW, Charleston B (2009) Foot-and-mouth disease virus can induce a specific and rapid CD4(+) T-cell-independent neutralizing and isotype classswitched antibody response in Naive Cattle. J Virol 83:36263636

McCahon D, Crowther JR, Belsham GJ, Kitson JD, Duchesne M, Have P, Meloen RH, Morgan DO, De Simone F (1989) Evidence for at least four antigenic sites on type $O$ foot-and-mouth disease virus involved in neutralization; identification by single and multiple site monoclonal antibody-resistant mutants. J Gen Virol 70(Pt 3):639-645

Pay TW, Hingley PJ (1987) Correlation of 140 S antigen dose with the serum neutralizing antibody response and the level of protection induced in cattle by foot-and-mouth disease vaccines. Vaccine 5:60-64

Paton DJ, Sumption KJ, Charleston B (2009) Options for control of foot-and-mouth disease: knowledge, capability and policy. Philos Trans R Soc Lond B 364:2657-2667
SaCarvalho D, Rieder E, Baxt B, Rodarte R, Tanuri A, Mason PW (1997) Tissue culture adaptation of foot-and-mouth disease virus selects viruses that bind to heparin and are attenuated in cattle. $J$ Virol 71:5115-5123

Qiu X, Lei Y, Yang P, Gao Q, Wang N, Cao L, Yuan S, Huang X, Deng Y, Ma W et al (2018) Structural basis for neutralization of Japanese encephalitis virus by two potent therapeutic antibodies. Nat Microbiol 3:287-294

Tuthill TJ, Groppelli E, Hogle JM, Rowlands DJ (2010) Picornaviruses. In: Cell entry by non-enveloped viruses. Springer, pp 43-89.

Wang N, Zhao D, Wang J, Zhang Y, Wang M, Gao Y, Li F, Wang J, Bu Z, Rao Z et al (2019) Architecture of African swine fever virus and implications for viral assembly. Science 366:640-644

Zhu L, Xu K, Wang N, Cao L, Wu J, Gao Q, Fry EE, Stuart DI, Rao Z, Wang $\mathrm{J}$ et al (2018) Neutralization mechanisms of two highly potent antibodies against human enterovirus 71 . MBio 9:66-69

Hu Dong and Pan Liu have contributed equally to this work.

Electronic supplementary material The online version of this article (https://doi.org/10.1007/s13238-021-00828-9) contains supplementary material, which is available to authorized users. 